\title{
Pilosebaceous Hamartoma
}

National Cancer Institute

\section{Source}

National Cancer Institute. Pilosebaceous Hamartoma. NCI Thesaurus. Code C5565.

A hamartoma characterized by localized pilosebaceous apparatus malformation. 